\title{
Detection of Vertical Charge Separation Current in a Toroidal ECR Plasma by Radially Aligned Multi-Electrodes
}

\author{
Kengoh KURODA, Masaki UCHIDA, Hitoshi TANAKA and Takashi MAEKAWA \\ Graduate School of Energy Science, Kyoto University, kyoto 606-8502, Japan
}

(Received 23 March 2012 / Accepted 23 May 2012)

\begin{abstract}
In plasmas immersed in the toroidal field $B_{\varphi}$, electrons drift downward while ions drift upward due to the field gradient and curvature $\left(B_{\varphi}>0\right.$ is assumed). The plasma is usually bounded at the top and bottom by the conducting vessel walls. The same amount of current must flow into and out from the walls at the top and bottom, respectively, to complete the current circulation via the vacuum vessel. In an ECR plasma in the LATE device radial profiles of vertical charge separation currents have been for the first time measured by radially aligned multi-electrodes fabricated at the top and bottom. Both the profiles at the top and bottom are nearly the same as the profile $2 P_{\mathrm{e}} / R B_{\varphi}$ in the plasma as theoretically predicted. Current characteristics upon the sweep of external voltage onto the top ion collectors reveal that the current is due to inflow of ions with no secondary electron emission.
\end{abstract}

(c) 2012 The Japan Society of Plasma Science and Nuclear Fusion Research

Keywords: ECR plasma, VTF drift, charge separation current, current circulation

DOI: $10.1585 /$ pfr.7.1302098

There has been considerable interest in toroidal electron cyclotron resonance (ECR) plasmas in open fields. For example, ECR pre-ionization is considered to be a useful method to assist the current startup of tokamak discharges [1, 2]. In addition ECR plasmas produced by microwaves, typically at $\sim 1 \mathrm{~kW}$ with a frequency of $2.45 \mathrm{GHz}$, in small devices have been frequently used as a useful bed for basic studies in various aspects of plasma physics including, for example, particle confinement [3,4], turbulence and blob generations [5,6]. While quite a large number of experiments have been done by using toroidal ECR plasmas as mentioned above, their equilibrium characteristics were hardly investigated except for a few studies [7-9].

Equilibrium of charged particles in a toroidal field $B_{\varphi}$ is governed by their vertical drift, here referred to as Vacuum Toroidal Field drift, with a velocity given by

$$
V_{Z}=\frac{m\left(v_{/ /}^{2}+v_{\perp}^{2} / 2\right)}{q R B_{\varphi}} .
$$

Electrons drift downward while ions drift upward $\left(B_{\varphi}>0\right.$ is assumed), generating a charge separation current. The local current density by electrons (ions) VTF drift is given by summing up the contribution of every electron (ion) over their Maxwell velocity distribution and is written in terms of local electron (ion) pressure $P_{\mathrm{e}}\left(P_{\mathrm{i}}\right)$,

$$
j_{\mathrm{Ze}(i)}=\frac{2 P_{\mathrm{e}}\left(P_{\mathrm{i}}\right)}{R B_{\varphi}} .
$$

The plasma is usually bounded at the top and bottom by the conducting vessel walls. Experimental observations

author'se-mail: kuroda.kengoh.65r@st.kyoto-u.ac.jp deny endless charge accumulation at the boundary as well as in the plasma, indicating that in a steady state the same amount of current must flow into and out from the walls at the top and bottom, respectively. The electrons that flow into the bottom wall would flow through the conducting vessel to the top wall on which they would recombine with the ions that are coming up from the plasma, completing the circulation as conjectured by S. Yoshikawa [10].

The current circulation was confirmed by recent experiments in the Low Aspect ratio Torus Experiment device, in which the circulation current via the vacuum vessel was detected by the combination of a panel and a limiter fabricated at the bottom and top for the collection of electrons and ions (see Fig. 1 in [8]). It was shown that both the currents flowing into the top and out from the bottom were the same and they were equal to the sum of the local electron VTF drift current over the horizontal cross section of the plasma. However, whether or not the radial profiles of inflow and outflow currents were the same as $2 p_{\mathrm{e}} / R B_{\varphi}$ was remained as a question.

More interesting question is what makes the two currents at the top and bottom boundaries balanced. Note that $j_{\mathrm{Ze}}$ is much larger than $j_{\mathrm{Zi}}$ in ECR plasmas, which would result in serious imbalance. There are two possibilities which may resolve this problem. First, a steep down-slope of space potential develops toward the top wall and the slope accelerates the ion VTF drift to the wall as proposed previously [9]. Another is the secondary electron emission upon the bombardment of the accelerated ions.

We have addressed above questions in the LATE device by using radially aligned multi-electrodes newly fabricated at the bottom and top of the vessel as shown in Fig. 1. 


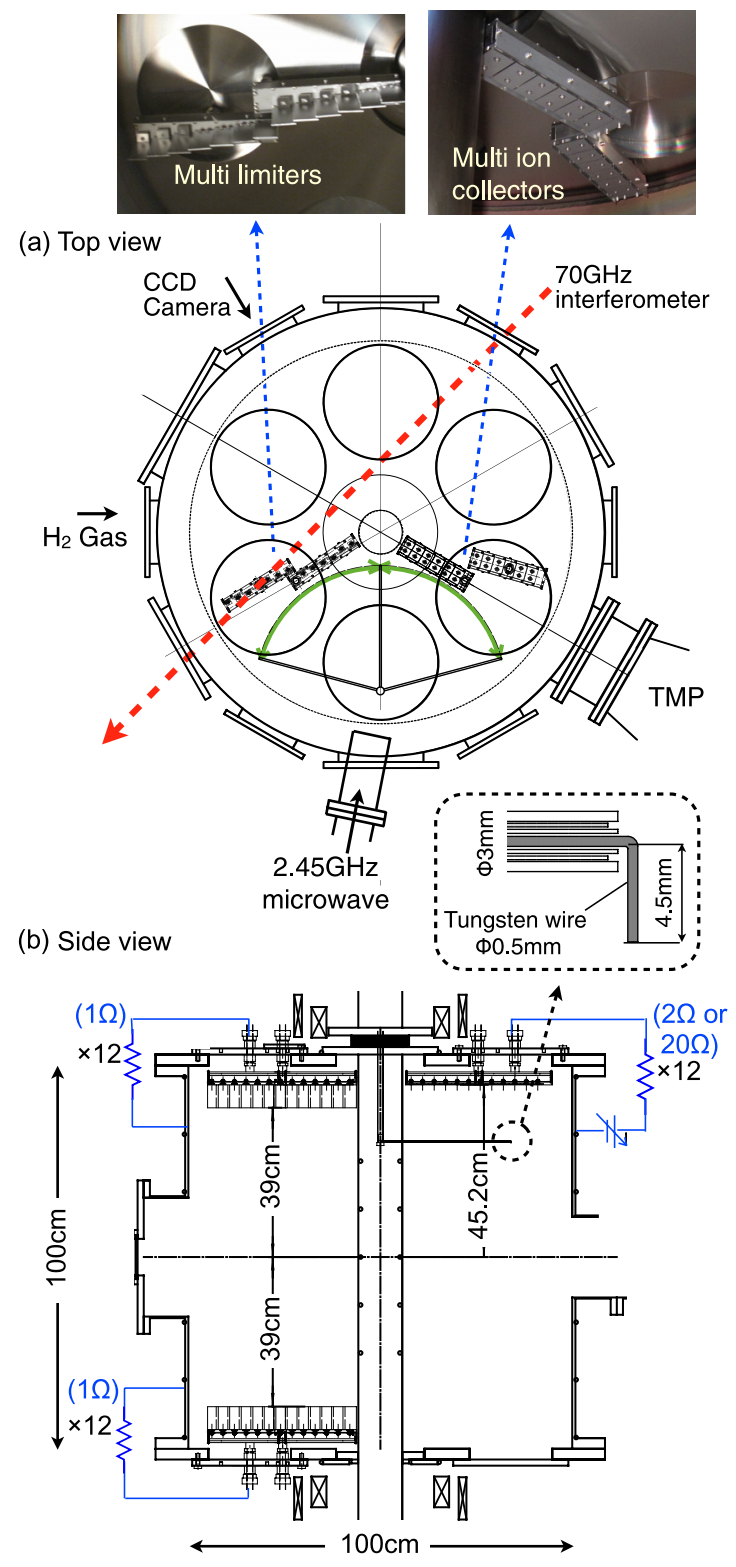

Fig. 1 Experimental apparatus.

Plasmas are generated by a $2 \mathrm{~s}, 1 \mathrm{~kW}$ microwave pulse at $2.45 \mathrm{GHz}$ under an initial filling hydrogen gas at $\sim 10^{-2} \mathrm{~Pa}$. The toroidal field is kept constant with the ECR layer at $R=13.7 \mathrm{~cm}$. The pressure decreases to $\sim 4 \times 10^{-3} P_{\text {a }}$ during initial $0.5 \mathrm{~s}$ with a decay time of $\tau \sim 0.25 \mathrm{~s}$ after the breakdown (the base pressure is $\sim 2 \times 10^{-4} \mathrm{~Pa}$ and the decay time without discharge is $\tau \sim 1.5 \mathrm{~s}$ ), and then it keeps this value until the end of the pulse under a weak continuous gas feeding. Both the line density along the horizontal chord on the mid-plane measured by a $70 \mathrm{GHz}$ interferometer and the plasma images on the CCD camera also become steady after initial $0.5 \mathrm{~s}$.

During the steady phase of $1.5 \mathrm{~s}$ after initial transient phase of $0.5 \mathrm{~s}$ various profiles are measured with a Langmuir probe inserted into the vessel from a top port as shown in Fig. 1. The probe is rotated by 37.5 degrees with a con- stant angular velocity around its vertical axis during this interval of $1.5 \mathrm{~s}$ and at the same time the probe voltage is swept sawtooth-likely many times to obtain the probe V-I characteristics at various radial locations. Four such rotations cover the whole range of angle depicted by green arc in Fig. 1 (a). For every Z-coordinates the same procedure of probe measurement has been done with a step of $1 \mathrm{~cm}$ from $Z=-24 \mathrm{~cm}$ to $48 \mathrm{~cm}$. Thus the probe tip covers the area from $R=8 \mathrm{~cm}$ to $41 \mathrm{~cm}$ and from $Z=-24 \mathrm{~cm}$ to $48 \mathrm{~cm}$ with a spatial resolution of $\sim 1 \mathrm{~cm}$, producing the profiles shown in Fig. 2.

The space potential $\left(V_{\mathrm{S}}\right)$ is distributed positively in the whole area of the upper side with a peak near the top as shown in Fig. 2 (a), which may convey the ions from the main part of plasma to the vicinity of top electrode as suggested previously [9]. The profiles of the electron density $\left(N_{\mathrm{e}}\right)$, temperature $\left(T_{\mathrm{e}}\right)$ and pressure $\left(P_{\mathrm{e}}\right)$ are quite different from $V_{\mathrm{S}}$. Roughly speaking both the $N_{\mathrm{e}}$ and $T_{\mathrm{e}}$ profiles have vertically extended ridges along the ECR layer. Near the top and bottom, however, the $N_{\mathrm{e}}$ profile somewhat spreads radially while the $T_{\mathrm{e}}$ profile somewhat shrinks radially. More remarkable is that at $Z=24-36 \mathrm{~cm}$ along the vertical ridges $T_{\mathrm{e}}$ increases while $N_{\mathrm{e}}$ decreases. Since the pressure is given by $P_{\mathrm{e}}=N_{\mathrm{e}} T_{\mathrm{e}}$, the excess and the deficit mentioned above compensate each other, making $P_{\mathrm{e}}$ profile quite uniform along $Z$ axis with a clear ridge along the ECR layer as shown in Fig. 2(d). This result may reflect the constraint $(\operatorname{div} \boldsymbol{j}=0$ ) on the vertical charge separation current (Eq. (1)).

The line integrated density by the $70 \mathrm{GHz}$ interferometer hardly depends on the position of the probe except for the initial transition time of discharge and coincides with the line-integrated value estimated from the probe results. The ion temperature along a vertical chord from $Z=30$ to $39 \mathrm{~cm}$ at $R=25 \mathrm{~cm}$ is measured to be $T_{\mathrm{i}} \sim 1 \mathrm{eV}$ by an ion sensitive prove [11] inserted from a top port, while $T_{\mathrm{e}} \sim 4 \mathrm{eV}$ along the same chord. Since $T_{\mathrm{i}}$ may be also much lower than $T_{\mathrm{e}}$ near the ECR layer, therefore ion contribution to the charge separation current may be negligible compared with electron contribution. In addition, since ion Lamour radius is quite small (less than $3 \mathrm{~mm}$ ) compared with the plasma size, the guiding center orbits by $\boldsymbol{E} \times \boldsymbol{B}$ drift are meaningful both for ions and electrons, and therefore, no net current is generated by the $\boldsymbol{E} \times \boldsymbol{B}$ drifts of ions and electrons. These results indicate that only the electron VTF drift current $j_{\text {Ze }}$ given by Eq. (1) flows in the main plasma.

We detect the vertical current by radially aligned multi-electrodes. Top electrode is a combination of multilimiters and multi-ion-collectors (see Fig. 1). Both the limiters and ion collectors are aligned radially with a period of $3 \mathrm{~cm}$ from $R_{\mathrm{CNTR}}=8 \mathrm{~cm}$ to $41 \mathrm{~cm}$, where $R_{\mathrm{CNTR}}$ denotes central position of each element. Radial width of limiters is $3.2 \mathrm{~cm}$. Radial width of ion collectors is $2.9 \mathrm{~cm}$ and their toroidal width is $4 \mathrm{~cm}$. The surface of ion collectors locates $6.2 \mathrm{~cm}$ higher than the limiter tip. The same multi-limiters 

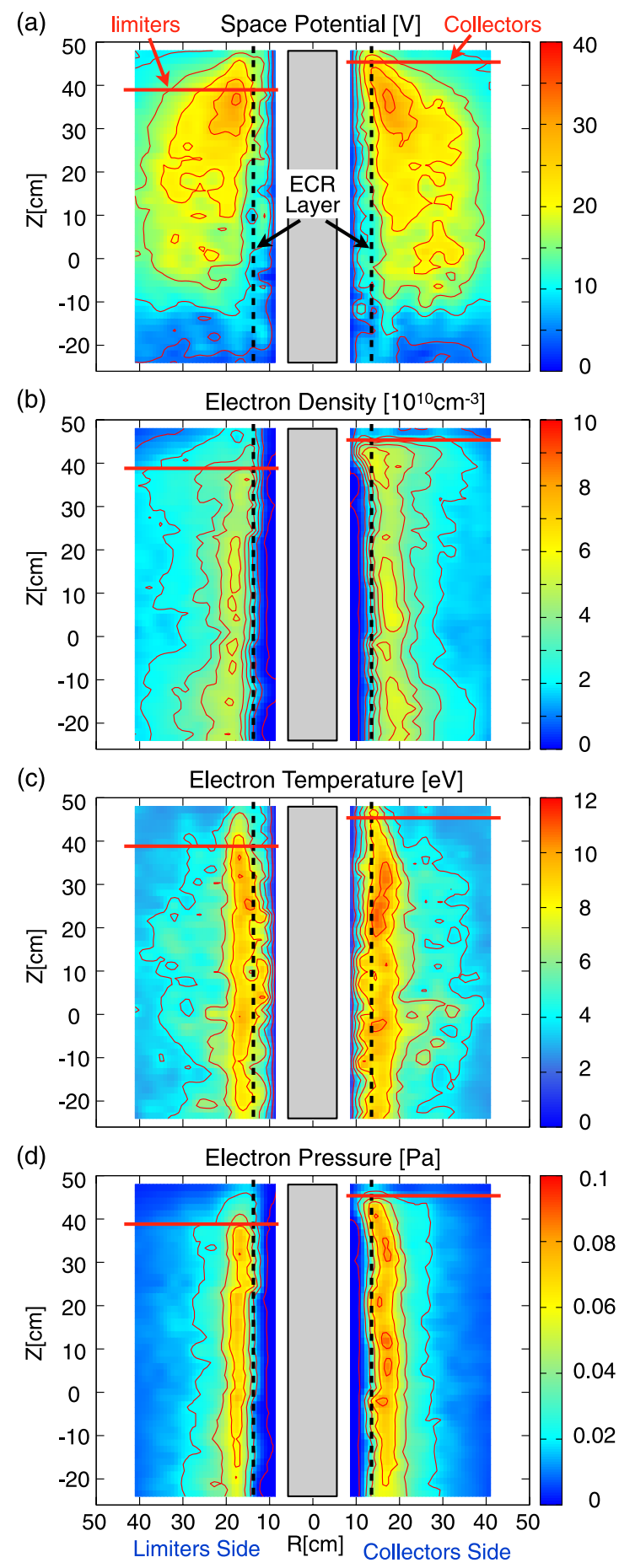

Fig. 2 Various plasma profiles.

are also fabricated at the bottom to detect the current from the bottom. Each electrode is electrically connected with the vessel through a low resistance to monitor the current between the electrode and the vessel. The sign of current is defined as positive for the direction to the electrode from the plasma.

Figure 3 shows voltage-current characteristics of multi-ion-collectors. They are obtained by applying a periodical sawtooth-like voltage from -40 to $40 \mathrm{~V}$ simultaneously to all the collectors. The collector at $R_{\mathrm{CNTR}}=17 \mathrm{~cm}$
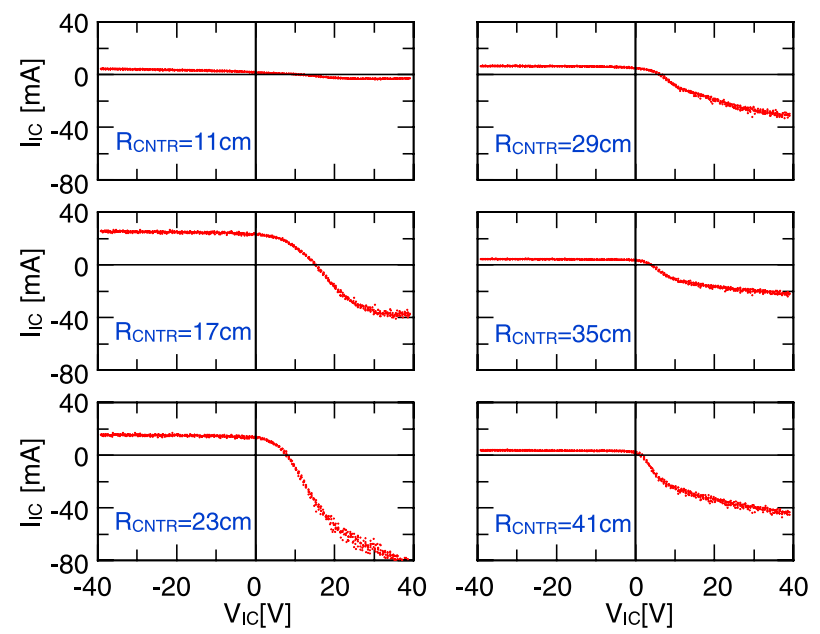

Fig. 3 V-I characteristics of ion collectors.

detects the largest ion current of $I_{\mathrm{IC}}=22 \mathrm{~mA}$ when the voltage relative to the vessel $V_{\mathrm{IC}}=0$. The characteristics indicate that when $V_{\mathrm{IC}}$ is increased positively electrons begin to flow in. The floating potential is $15 \mathrm{~V}$, which means that the collector collects ions at least up to $V_{\mathrm{IC}}=15 \mathrm{~V}$. These ions may reach the collector with kinetic energies more than $15 \mathrm{eV}$ when $V_{\mathrm{IC}}=0$. These energies are much higher than the ion temperature of $1 \mathrm{eV}$ in the main plasma. This large enhancement of kinetic energy is consistent with the model of the potential slope acceleration of VTF drift [9]. Note that there is a steep potential slope near the collector (Fig. 2(a)). Another important point is that ion current does not change when the collector voltage is decreased from $V_{\mathrm{IC}}=0$ to $-40 \mathrm{~V}$, indicating that the current is essentially due to the incoming ions and there is no electron contribution including both incoming electrons from the plasma and outgoing electrons due to the secondary electron emissions. The other collectors than $R_{\mathrm{CNTR}}=17 \mathrm{~cm}$ have nearly the same characteristics.

Figures 4 (b) and (d) show the radial profiles of the vertical currents detected by the ion-collectors, the top limiters and the bottom limiters, respectively. In these figures, the data are plotted in terms of current density defined as follows. In the case of ion collector currents, the current density is calculated by $j_{\mathrm{IC}}=I_{\mathrm{IC}} / S, S=4 \times 2.9 \mathrm{~cm}^{2}$ is the collector area. In the case of the limiters, the current density is calculated by $j_{\mathrm{LMT}}=I_{\mathrm{LMT}} / S_{\mathrm{ANNL}}$, where $S_{\mathrm{ANNL}}=2 \pi R_{\mathrm{CNTR}} \Delta R$ is the area of annular with the limiter radial width of $\Delta R=3 \mathrm{~cm}$ and $I_{\mathrm{LMT}}$ is the limiter current. Note that $j_{\mathrm{LMT}}$ at the top limiter is quite smaller than that at the bottom limiter [9].

Figure 4 (c) shows radial profiles of the electron VTF drift current density given by Eq. (1) for radial profiles of electron pressure at various vertical locations at the ioncollector side. Those at the limiter side are nearly the same. All the radial profiles of vertical charge separation currents in Figs. 4 (b), (c) and (d) are nearly the same except that the 


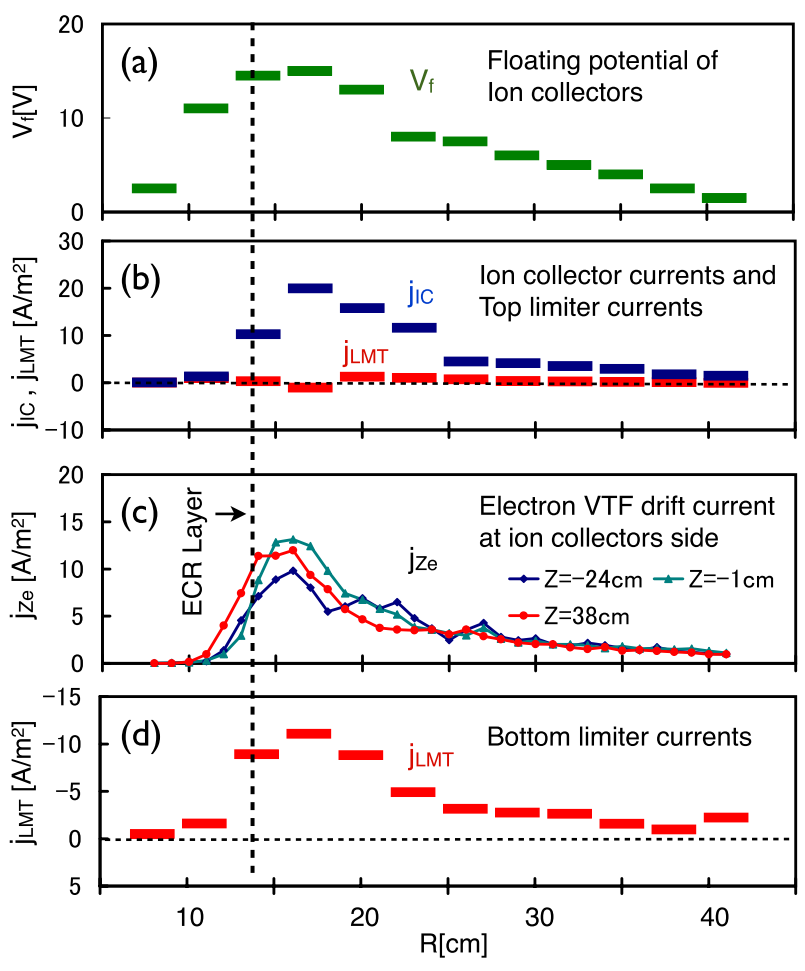

Fig. 4 Radial profiles of floating potential of ion collectors and vertical charge separation currents at the top, in the plasma and at the bottom.

$j_{\text {IC }}$ profile in (b) is 1.7 times larger than the $j_{\mathrm{LMT}}$ profile in (d).

In the previous experiment in which a full panel covered the whole plasma from top, however, the panel current was the same as the bottom limiter current $[8,9]$. There may be a factor which enhances the current into present local ion collectors as shown in Fig. 5. The profile of space potential just below the ion collectors is different from those in Fig. 2 (a). $V_{\mathrm{S}}$ must be zero at the surface of ion collectors. Such a 3D $V_{\mathrm{S}}$ profile may attract ions toward the ion collectors from both toroidal sides when the ions are VTF drifting up toward the collector. When we take into account the previous result and the factor described above, the results in Figs. 4 (b), (c) and (d) suggest that the vertically aligned charge separation current driven by the VTF drift of EC-heated electrons flows out from the bottom wall and flows into the top wall with keeping its radial profile.

While the vertical current is essentially driven by the VTF drift of electrons in the main plasma, the current carrier is replaced from electrons to ions just before the top boundary, where a steep down-slope of space potential develops toward the top wall as shown in Fig. 2 (a) and the energy of the ions becomes as high as the electron thermal energy when they descend down to the collector as suggested by the floating potential of ion collectors shown in

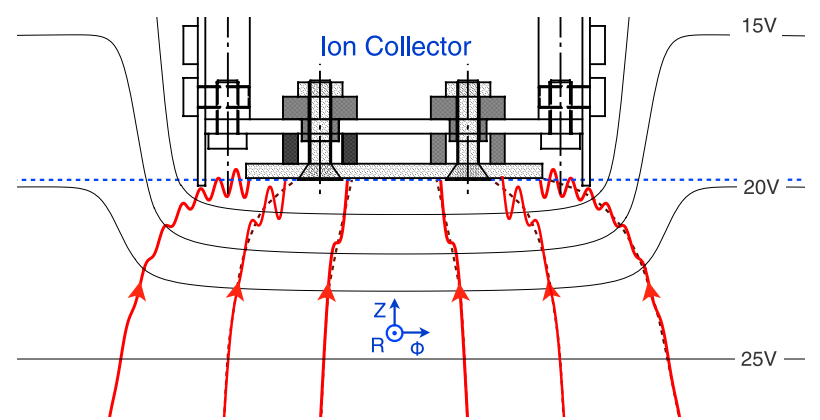

Fig. 5 Ions are collected from wider area than the collector area due to the effect of a 3D electrostatic potential developed around the collector.

Fig. 4 (a), being consistent with the model of the steep potential slope acceleration of VTF drift of ions [9].

In summary, in an ECR plasma in the LATE device the boundary profiles of vertical charge separation current driven by the VTF drift of EC heated electrons have been for the first time measured by radially aligned multielectrodes fabricated at the top and bottom. Both the current profiles at the top and bottom are nearly the same as the electron VTF drift profile of $2 P_{\mathrm{e}} / R B_{\varphi}$ in the plasma as theoretically predicted. Current characteristics upon the sweep of external voltage on the ion collectors reveal that the current is due to inflow of ions with no secondary electron emission. In addition the energy of the ions becomes as high as the electron thermal energy, suggesting that the potential slope acceleration of VTF drift generates the observed ion current at the boundary.

The present work was supported by Grants-in-Aid scientific research from JSPS and NIFS collaboration program in Japan.

[1] J. Stober et al., Nucl. Fusion 51, 083031 (2011).

[2] K. Kajiwara Y. Ikeda, M. Seki, S. Moriyama, T. Oikawa and T. Fujii, Nucl. Fusion 45, 694 (2005).

[3] S. Nakao, K. Ogura, Y. Terumichi and S. Tanaka, Phys. Lett. A 96, 405 (1983).

[4] S.H. Müller et al., Phys. Rev. Lett. 93, 165003 (2004).

[5] H. Zushi et al., in 22nd IAEA Fusion Energy Conference, (11-16, Oct. 2010, Daejeon, Korea) EXS/P2-22.

[6] I. Furno et al., Phys. Rev. Lett. 100, 055004 (2008).

[7] L.E. Zakharov and G.V. Pereverzev, Sov. J. Plasma Phys. 14, 75 (1988).

[8] S. Nishi, T. Sakabe, M. Uchida, H. Tanaka and T. Maekawa, Plasma Phys. Control. Fusion 52, 065011 (2010).

[9] S. Nishi, T. Sakabe, M. Uchida, H. Tanaka and T. Maekawa, Plasma Phys. Control. Fusion 52, 125004 (2010). See sections 4.4 and 4.5.

[10] S. Yoshikawa, W.I. Harries and R.M. Sinclair, Phys. Fluids 6, 1506 (1963).

[11] N. Ezumi et al., J. Nucl. Mater. 337-339, 1106 (2005). 